\title{
A Model for the Elasticity of Compressed Emulsions
}

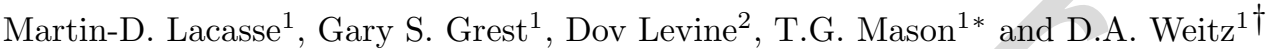 \\ ${ }^{1}$ Corporate Research Science Laboratories \\ Exxon Research and Engineering Co., Annandale, NJ 08801 \\ ${ }^{2}$ Department of Physics, Technion, Haifa, 32000 Israel
}

(December 13, 1995)

\begin{abstract}
We present a new model to describe the unusual elastic properties of compressed emulsions. The response of a single droplet under compression is investigated numerically for different Wigner-Seitz cells. The response is softer than harmonic, and depends on the coordination number of the droplet. Using these results, we propose a new effective inter-droplet potential which is used to determine the elastic response of a monodisperse collection of disordered droplets as a function of volume fraction. Our results are in excellent agreement with recent experiments. This suggests that anharmonicity, together with disorder, are responsible for the quasi-linear increase of $G$ and $\Pi$ observed at $\varphi_{c}$.

PACS numbers: 82.70.Kj, 81.40.Jj, 62.20.Dc
\end{abstract}

Emulsions are materials with highly unusual elastic properties. They consist of droplets of one fluid dispersed in a second fluid, with interfaces stabilized by a surfactant. Despite being comprised solely of fluids, they can be elastic solids, when droplets are compressed to a large volume fraction, $\varphi$, by an osmotic pressure, $\Pi$. The origin of this elasticity is the interfacial energy of the droplets. At low volume fractions, their surface tension, $\sigma$, ensures that the droplets are spherical in shape; however, at higher $\varphi$, the packing constraints force the droplet shapes to deform, storing energy. The application of a shear strain to a compressed emulsion causes the droplets to deform further, increasing their surface area, thereby storing elastic energy [1, 2, 3, 他, 5].

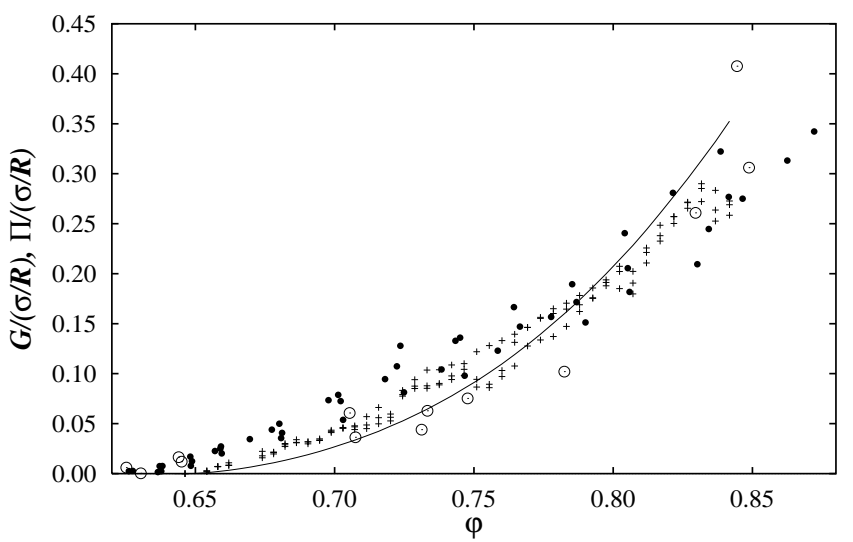

FIG. 1. Experimental values of the osmotic pressure $(\odot)$ and elastic shear modulus $(\bullet)$ of different emulsions with monodisperse droplets. The data are normalized by $\sigma / R$, allowing data from emulsions with different droplet sizes to be compared. Results predicted by the present model for the shear modulus $(+)$ and the osmotic pressure (line) are also shown.

The experimentally measured [6,7] $\varphi$ dependence of the static shear modulus $G$ is remarkable in several ways. Typical data [7] obtained for monodisperse [8] siliconeoil-in-water emulsions are summarized in Fig. 1. The solid symbols show $G$ measured as a function of $\varphi$ for several monodisperse emulsions of different droplet sizes. The data are normalized by $\sigma / R$ where $R$ is the radius of an undeformed droplet. The observed scaling by the Laplace pressure $(2 \sigma / R)$ confirms the essential role of the interfacial energy. The magnitude of the scaled modulus increases approximately linearly, varying as $G \sim \varphi\left(\varphi-\varphi_{c}\right)$, where $\varphi_{c}$ is the volume fraction where the droplets are first deformed. For monodisperse droplets, $\varphi_{c} \approx 0.64$, the maximum volume fraction that monodisperse spheres can be randomly packed [7,9]; for polydisperse droplets $\varphi_{c}$ is larger [6, reflecting more efficient packing. Surprisingly, the osmotic pressure required to compress the emulsion is very similar to the shear modulus [7, 10], as shown by the open circles in Fig. 1. This implies that the corresponding longitudinal bulk elastic modulus, or the bulk osmotic modulus, $K=\varphi d \Pi / d \varphi$, must differ significantly from the shear modulus as it must have a much sharper onset at $\varphi_{c}$ [7].

The behavior of the shear modulus of emulsions has been the subject of various theoretical studies. A first approach consists in considering a single droplet, and to obtain the properties of a periodic structure, assuming the strain remains affine [1, 何, 河. While this can be done analytically in two dimensions, in three dimensions it can only be done through approximations. Results obtained from this approach consistently predict a sharp rise of $G$ at $\varphi_{c}$, followed by a much slower increase at larger $\varphi$. This suggests that the effects of disorder may be responsible for the quasi-linear rise of $G$ at $\varphi_{c}$ found experimentally. The response to shear of disordered arrangements of droplets has also been investigated. However, due to the additional complexity this imposes, these calculations have been restricted to two dimensions [2, 3, 11, 12], where disorder needs to be introduced through polydispersity. Nevertheless, these two-dimensional models show a different behavior than that observed experimentally for disordered, three-dimensional emulsions. In particular, two-dimensional systems do not exhibit a smooth nearly linear increase of $G$ near $\varphi_{c}$, although a smooth quadratic increase of $\Pi$ near $\varphi_{c}$ has recently been observed [11]. 
In this Letter, we present the first three-dimensional computer simulation modeling the elasticity of a disordered emulsion. In addition, we propose a new, more realistic inter-droplet potential. It is based on numerical results obtained by calculating the change in surface energy of a single droplet as it is compressed using Brakke's Surface Evolver (SE) software [13]. Our model captures the essential physics of the droplet interactions and their disordered, glassy packing. The droplets are soft, monodisperse spheres interacting through a purely repulsive potential. The droplet positions are allowed to relax upon application of shear. We calculate the osmotic pressure and the shear modulus as a function of volume fraction. We find that both the positional relaxation of the droplets and the form of the potential are essential in reproducing the experimental behavior of the shear modulus.

We first investigate in more detail the interaction between droplets. When two droplets are forced together, their spherical shapes are deformed and their surfaces develop flattened facets at contact. As a simplification, consider a droplet of radius $R$ compressed between two parallel planes, each one located at a distance $h$ from the center of the droplet. Naively, for small deformations $\delta \xi$, where $\xi=(R-h) / R$ is a dimensionless measure of compression, the resultant force, $F$, on the flat facets can be estimated by assuming that the radius of the droplet, and hence the Laplace pressure, remains unchanged. We thus have $F \approx(2 \sigma / R) \delta S$, where $\delta S$ is the area of the flattened facet. To linear order in the deformation, $\delta S \approx 2 \pi R^{2} \delta \xi$, so that $F \approx 4 \pi \sigma R \delta \xi$. Thus, the interaction between the facets of two droplets is often taken as a strictly repulsive harmonic spring of spring constant $4 \pi \sigma$ acting between the centers of the spheres [3, 12].

The harmonic-spring potential, while appealing, ignores the details of the response of the shape to deformation [4, 14, and the possibility of coupling between the different facets on each droplet. Thus, to determine an improved potential, we investigated numerically the shape of a single droplet confined within space-filling polyhedral cells. Using SE we calculated the excess surface as the confinement is increased, under the constraint of a fixed droplet volume. As confining cells we investigated a rhombic dodecahedron (f.c.c.), a truncated octahedron (b.c.c.) and a simple cube (s.c.). In all cases, the compressions leave the center of mass unchanged, so that a distance $h$ from the center can be defined.

For simplicity, we shall define $\sigma=1$, and $R=1$. For a droplet of total surface area $A$, we can thus define the excess energy as $E_{1}=(A-4 \pi)$. Figure 2 shows the calculated dependence of the excess energy per facet, $E_{1} / n$, on $\xi$ for different types of cells, where $n$ represents the number of facets. The data are well described by $\left[(R / h)^{3}-1\right]^{\alpha}$ as shown by the dashed lines. For small $\xi$, this form reduces to a simple power law, $(3 \xi)^{\alpha}$, as is evidenced in Fig.2 by the asymptotically linear behavior. This func-

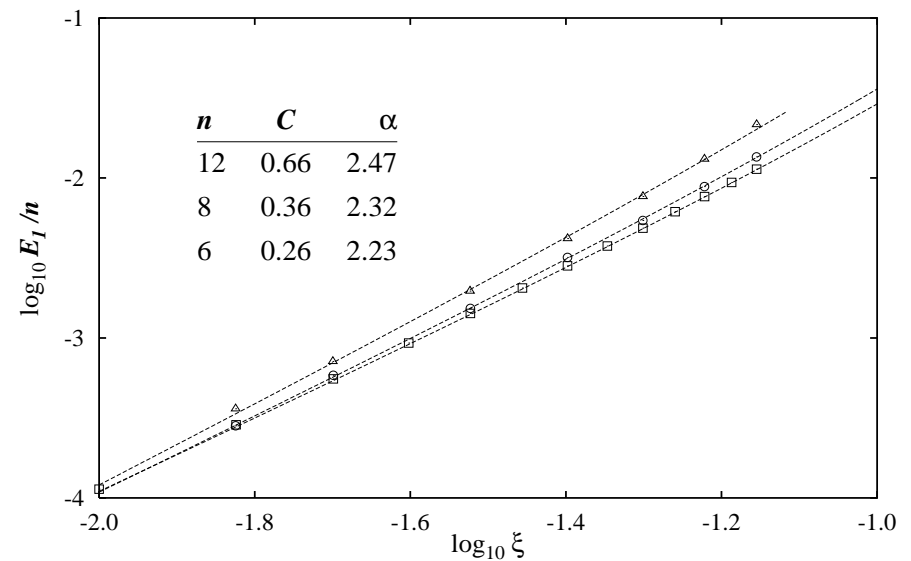

FIG. 2. Excess surface energy excess per facet under different Wigner-Seitz constraining cells. The lines are obtained from a fit to $E_{1} / n=C\left(\varphi / \varphi_{c}-1\right)^{\alpha}$, where $\varphi / \varphi_{c}=(R / h)^{3}=1 /(1-\xi)^{3}$. Curves are, from top to bottom: f.c.c. $\left(n=12, \varphi_{c}=\pi \sqrt{2} / 6\right)$, b.c.c. $\left(n=8, \varphi_{c}=\pi \sqrt{3} / 8\right)$, and s.c. $\left(n=6, \varphi_{c}=\pi / 6\right)$. Values of $C$ and $\alpha$ are accurate to 0.05 .

tional form is also equivalent to $\left(\varphi-\varphi_{c}\right)^{\alpha}$ for space-filling structures. Fitting to $E_{1} / n=C\left[(R / h)^{3}-1\right]^{\alpha}$, we find that both the coefficient, $C$, and the exponent, $\alpha$, depend on $n$, as is evident from the data in Fig 2. Their values are shown in the table inserted in Fig. 2. The data selected for the fits lie in the $\xi$ interval $0-7 \%$, the upper value corresponding to $\varphi=0.92$ for an f.c.c. lattice. For the b.c.c. lattice we use $n=8$ since second neighbors do not contribute to the energy for $\varphi \lesssim 0.90$ [14]. Our results show explicitly that the response of a droplet to compression is a non-local phenomenon: the response depends on the number of planes used to compress the droplet. One might expect that it also depends on the relative orientation of these planes. However, comparison between results obtained for iso- $n$ configurations, for instance, the pentagon dodecahedron and the rhombic dodecahedron, suggests that the distribution of the compressing planes on the droplet surface has only a minor effect [14]. Thus, we expect a similar functional form for $E_{1}$, even when the planes are more randomly distributed as they would in a disordered emulsion.

This behavior is different from the one encountered in two-dimensional systems. A minimum free surface is characterized by a uniform pressure or, equivalently by a uniform mean curvature. In two dimensions, the surface is parameterized by only one radius of curvature and the minimum free surface is always an arc of a circle. In this case, a harmonic potential is a good approximation. By contrast, in three dimensions, the response is always softer than harmonic since $\alpha(n)>2$ for all values of $n$ investigated here; thus, the effective spring constant goes to zero as the distortion vanishes. We note that, as proposed, a $n$-dependent power-law leads to unphysical behavior for very small compressions, as the energies for 
different $n$ should cross. However, this effect is too small in magnitude to affect our results. The functional form we use is a convenient way to mimic the response of a droplet over the range of compression we investigate.

To describe the elastic properties of a disordered droplet packing, we use a model which replaces the droplets by soft spheres which interact with their nearest neighbors through central-force potentials that reflect the behavior of the facets. However, since we found that the energy curve of each contact is a function of the droplet coordination number, the interaction energy should be obtained by balancing the forces at each contact, using the values of $n$ for each droplet. To make the computation tractable, we determine the average coordination number, $\bar{n}$, of the whole configuration of droplets, and use a single effective potential for that configuration. This is a reasonable approximation given the rather narrow distribution of $n$ for our monodisperse systems. Thus, we use the SE results to define a repulsive, central-force inter-droplet potential,

$$
U(d)= \begin{cases}2 C\left[\left(\frac{2 R}{d}\right)^{3}-1\right]^{\alpha}, & (d<2 R) \\ 0, & (d \geq 2 R)\end{cases}
$$

where $d=2 h$ is the distance between the droplet centers, and the factor 2 accounts for the two facets on the interacting pair. During the simulation, the exponent $\alpha(\bar{n})$ and the prefactor $C(\bar{n})$ are estimated from cubic interpolations of the values shown in Fig.2. The value of $\bar{n}$ is observed to increase from $\bar{n}_{c} \approx 6$ at $\varphi_{c} \approx 0.64$ to $\bar{n} \approx 10$ at $\varphi=0.84$. We finally note that central-force potentials can only include compressional distortion; nevertheless we use them to describe shear distortions as well.

The relaxation algorithm entails the minimization of the $3 \mathrm{~N}$-dimensional function,

$$
E_{N}=\sum_{i, j>i}^{N} U\left(d_{i j}\right)
$$

where $d_{i j}$ is the distance between point-particles $i$ and $j$. We use a conjugate-gradient (CG) method coupled to Brent line-minimization method [15]. This process represents a damped relaxation of a system of interacting droplets, and therefore the masses of the particles are not necessary. In order to find only a local minimum, our version of CG is written so that each lineminimization search interval is defined using the result of the line-minimization along the previous conjugate direction. The evaluation of the potential benefits from techniques borrowed from molecular dynamics, such as a Verlet table [16]. The algorithm is designed to return after a minimum number of iterations and when new values for the energy differ by less than $\delta E_{N} / E_{N} \leq 10^{-7}$. For each relaxed configuration, the energy, the average coordination number, and the pressure are measured. The coordination number is derived from the sum of all pairs contributing at least a small $\epsilon$ to the energy and values for the pressure in each Cartesian direction (diagonal elements of the stress tensor) are obtained from the virial 16.

We first construct a random distribution of $N$ monodisperse particles of radius $R$ in a cubic container with periodic boundary conditions. Typical runs start from a configuration prepared at the desired volume fraction. Systems that were slowly compressed from $\varphi<\varphi_{c}$ to $\varphi>\varphi_{c}$, and random configurations built and relaxed at $\varphi>\varphi_{c}$ were found to have similar elastic properties. Thus, we usually start at $\varphi \approx 0.67>\varphi_{c}$ in order to avoid the long computational time required to relax a configuration at $\varphi_{c}$. The system is then compressed and relaxed in small step increments up to $\varphi \approx 0.84$. From this value, the cubic container is sheared using isochoric uniaxial strains wherein, say, the $z$ axis is stretched by a factor $\lambda \gtrsim 1$ and the perpendicular plane is compressed by $\lambda^{-\frac{1}{2}}$. The shear modulus is obtained from the excess energy density as a function of the extension ratio $\lambda[17]$,

$$
E_{N} / V=E_{N}^{o} / V+\frac{1}{2} G\left(\lambda^{2}+2 / \lambda-3\right)
$$

where $E_{N}^{o}$ is the excess energy of the unstrained system. We perform several strain cycles to allow for relaxation and to verify reproducibility. This shearing process is performed along the three Cartesian directions. The system is then expanded in a small step increment, relaxed, and the shearing procedure is repeated. System sizes of at least $N=10^{3}$ were used to avoid undesired relaxation into an f.c.c. structure at large strains.

The calculated $\varphi$ dependence of the shear modulus is shown by the plus symbols in Fig. 11, while the osmotic pressure is shown by the solid line, for a system of $N=10^{3}$. Interestingly, the data for $\Pi$ shows selfaveraging and contains much less fluctuations that that for $G$. This suggests that there is an effective larger correlation length that determines the modulus and limits the averaging possible in our finite systems. Nevertheless, remarkably good agreement with the experimental data is obtained. Both the magnitudes and the $\varphi$ dependencies are correctly reproduced for both $G$ and $\Pi$.

The simulation also provides physical insight about the origin of the behavior of the shear modulus of emulsions. There are two essential effects. The first is the positional relaxation of the droplets. We illustrate this graphically in Fig. 3: we subtract the actual motion of the droplets from the affine motion caused by a strain associated with $\lambda=1.0006$, and use arrows at the center of each droplet to signify the direction and magnitude of this difference. The length of each arrow has been increased by 250 to make the motion with such a small strain observable. The droplet motion is clearly not affine; moreover, the difference appears random in direction. The importance of this non-affine motion is reinforced by calculating the shear modulus for a strictly affine (unrelaxed) motion; it 


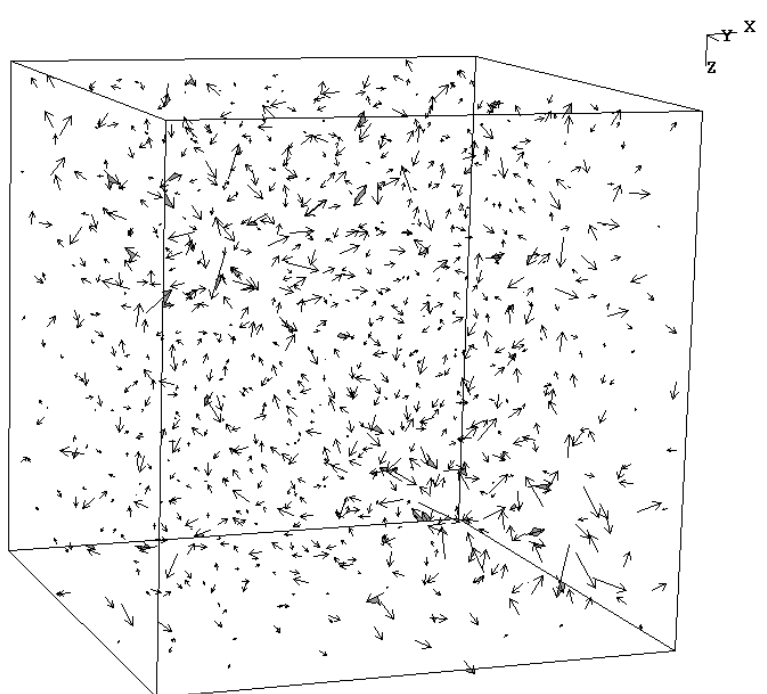

FIG. 3. An isochoric uniaxial shear strain of $\lambda=1.0006$ is applied to a $N=10^{3}$ system. Each arrow, magnified 250 times, represents the droplet relaxation from an affine, non-relaxed strain. $\varphi=0.80$.

increases by a factor of about 3 for all $\varphi$. We emphasize, however, that the non-affine motion of the droplets does not result in large scale rearrangements of their positions. We found that only a very small fraction of the droplets change neighbors during shear. Thus, the non-affine motion results from localized relaxation of the droplet positions [18]. The second essential feature is the anharmonicity of the potential. We performed similar simulations using a harmonic potential and obtained qualitatively different results 19] for $G$, which exhibited a significantly steeper rise near $\varphi_{c}$, similar to what is observed in harmonic two-dimensional systems [3, 12]. By contrast, the behavior of $\Pi$, is not as sensitive to the potential.

Finally, we note that the average coordination number was found to increase continuously above $\varphi_{c}$, consistent with a power law increase $\left(\bar{n}-\bar{n}_{c}\right) \sim\left(\varphi-\varphi_{c}\right)^{\frac{1}{2}}$ surprisingly the same functional form as the one observed in computer simulations in two dimensions [12]. In the present picture, the increase of $\bar{n}$ not only creates more contacts capable of storing energy, but also causes the energy of the existing contacts to increase because of the increase of the number of facets around each droplet. However, this has only a minor effect as confirmed by simulations using fixed $C$ and $\alpha$.

These results suggest an explanation for the origin of the surprising elasticity of emulsions; moreover, they will likely have broader significance. They are immediately applicable to foams, since the compressibility of the gas in the bubbles is typically much less than the Laplace pressure. More generally, emulsions are an example of a material with strictly repulsive interactions which is nevertheless a solid when confined by an osmotic pressure. The elasticity of these packings is significantly different than that of more traditional materials, and the results presented here should form the basis for developing a more comprehensive description of these fascinating materials.

We thank Shlomo Alexander for useful discussions, Le Fonds FCAR du Québec and the U.S.-Israel Binational Science Foundation for financial support.

* Present Address: Centre de Recherche Paul Pascal, Ave A. Schweitzer, F-33600 Pessac, France.

$\dagger$ Present Address: Dept. of Physics and Astronomy, University of Pennsylvania, Philadelphia, PA 19104.

[1] H.M. Princen, J. Colloid Interface Sci. 91, 160 (1983).

[2] A.M. Kraynik, Ann. Rev. Fluid Mech. 20, 325 (1988).

[3] F. Bolton and D. Weaire, Phys. Rev. Lett. 65, 3449 (1990).

[4] D.C. Morse and T.A. Witten, Europhys. Lett 22, 549 (1993); D.C. Morse, private communication.

[5] D.M.A. Buzza and M.E. Cates, Langmuir 10, 4503 (1994).

[6] H.M. Princen and A.D. Kiss, J. Colloid Interface Sci. 112, 427 (1986).

[7] T.G. Mason, J. Bibette and D.A. Weitz, Phys. Rev. Lett. 75, 2051 (1995).

[8] J. Bibette, J. Colloid and Interface Sci. 147, 474 (1991).

[9] J.G. Berryman, Phys. Rev. A 27, 1053 (1983) and references therein.

[10] H.M. Princen and A.D. Kiss, Langmuir 3, 36 (1987).

[11] S. Hutzler and D. Weaire, J. Phys.: Condens. Matter 7, L657 (1995).

[12] D. Durian, Phys. Rev. Lett. 75, 4780 (1995).

[13] K. Brakke, Exp. Math. 1, 141 (1992).

[14] M.-D. Lacasse, G.S. Grest, and D. Levine, in preparation.

[15] G. Forsythe, M. Malcolm and C. Moler, Computer methods for mathematical computations (Mir, Moscow, 1980).

[16] M.P. Allen and D.J. Tildesley, Computer simulation of liquids (Clarendon Press, Oxford, 1987).

[17] L.R.G. Treloar, Rep. Prog. Phys. 36, 755 (1973).

[18] A.J. Liu, S. Ramaswamy, T.G. Mason, Hu Gang and D.A. Weitz, in press.

[19] M.-D. Lacasse, G.S. Grest, D. Levine and D.A. Weitz, in preparation. 